\title{
Las repercusiones de la programación de los equipamientos culturales de Santiago de Chile en su entorno urbano
}

Daniel Paul i Agusti'; dpaul@geosoc.udl.cat

\section{RESUMEN}

El incremento de funciones que los equipamientos culturales han experimentado en los últimos años los ha transformado en elementos de primer orden de las políticas urbanas. A pesar de ello existen relativamente pocos estudios referentes a las repercusiones que estos espacios generan en sus entornos físicos, sociales y económicos. El artículo analiza estas repercusiones en el caso de los equipamientos culturales de Santiago de Chile. A partir del estudio de los equipamientos culturales y de las distintas interacciones existentes con el entorno urbano se apunta que en la actualidad los equipamientos analizados realizan un importante esfuerzo en el campo de la programación. Un esfuerzo que pocas veces se corresponde con una voluntad de difusión. Ello supone unas repercusiones limitadas de los equipamientos culturales en el entorno urbano. Una situación que obliga a una reflexión, especialmente en un contexto, el chileno, de incremento del número y ambición de equipamientos culturales.

Palabras clave: Política cultural; equipamientos culturales; Santiago de Chile

\section{The impact of new cultural facilities on the creative quarters of Santiago de Chile}

\begin{abstract}
The increase in functions that cultural facilities have undergone in recent years has transformed them into leading elements in urban development policies. Even so, relatively few studies make reference to the repercussions that these spaces generate in their physical, social and economic environments. The article will analyze these repercussions in the case of the cultural facilities of Santiago de Chile. We analyzed several aspects related to the activities of the cultural facilities. The data obtained show a significant effort in conducting these activities. However, this is an effort that rarely corresponds to a will for diffusion. This implies a number of limited repercussions for cultural facilities in the urban environment. It is a situation which calls for reflection, especially within a context, like that of Chile, with an increase in the number and objectives of cultural facilities.
\end{abstract}

Keywords: Cultural policy; cultural facilities; Santiago de Chile

Recibido el 31 de mayo de 2015, aceptado el 5 de julio de 2015.

1 Departamento de Geografía y Sociología, Universitat de Lleida, Plaça Víctor Siurana, 125003 Lleida, España. 


\section{INTRODUCCIÓN}

Las funciones de los equipamientos culturales han evolucionado enormemente a lo largo de los años. Se ha pasado de unos equipamientos pensados básicamente como escaparates de las distintas artes, a espacios dedicados a tareas mucho más amplias. Así, progresivamente, los distintos espacios culturales han ido incorporando funciones de estudio, de difusión y de creación de contenidos. En este contexto los equipamientos culturales se han transformado, por ejemplo, en importantes focos de atracción turística. En otros casos, han liderado la imagen de ciertas transformaciones urbanas. Así mismo, varias instituciones culturales se han incorporado en proyectos educativos y sociales fundamentales para la cohesión social de ciertas áreas. Se trata, en consecuencia, de un cambio profundo de las actividades de los equipamientos culturales (NAVARRETE 2008; DE GRAAFF et al. 2009). Unos equipamientos que ya no pueden ser analizados únicamente por sus actividades internas, sino que deben analizarse de una forma mucho más amplia y abierta a la sociedad.

Los equipamientos cultuales se han transformado en un elemento de primer orden de las distintas políticas urbanas. En este contexto, han sido muchos los gobiernos locales que han apostado por la creación o modernización de equipamientos culturales, lo que ha alterado el panorama tradicional. Ahora bien, este proceso generalmente no ha sido fruto de un consenso con los distintos agentes sociales y económicos de las ciudades (EVANS \& FOORD 2008). Generalmente no ha existido un debate profundo sobre las fortalezas y las debilidades que las nuevas funciones podían generar, lo que se ha traducido en cierta pérdida de los eventuales beneficios derivados de algunas de las actividades. Así, en cierto punto, podría existir una sobreoferta de actividades o un enfoque incorrecto que llevasen a equipamientos o actividades sin las repercusiones deseables en su entorno.

En este contexto, el artículo analizará las repercusiones de las distintas actividades de los equipamientos culturales en campos como las repercusiones económicas, culturales, sociales o urbanísticas. Para ello se analizan un conjunto de 28 equipamientos culturales de Santiago de Chile, buscando interrelacionar el volumen de actividades desarrollado por dichos equipamientos con los cambios que generan en el espacio y las repercusiones obtenidas a más largo plazo.

\section{Los equipamientos culturales en el contexto urbano.}

Las relaciones entre actividad artística y espacio urbano han sido una constante. Des de los años setenta se ha analizado los cambios que tuvieron, especialmente para los espacios centrales, la instalación de artistas, galerías y salas de exposiciones (BIANCHINI \& PARKINSON 1993; BARKE \& HARROP 1994; KEMP 2004). Así mismo, a partir de los años ochenta y especialmente de los noventa, se empieza a analizar el rol de las actividades culturales como motor de la transformación de los centros históricos de las ciudades europeas (EVANS 2001; GARCÍA 2004; PONZINI 2009). De esta época sobresalen proyectos como por ejemplo el Gran Louvre en París o la creación del museo Guggenheim de Bilbao.

El éxito de estos y otros proyectos supusieron un salto importante para la inclusión de las actividades culturales en distintos campos. Los equipamientos culturales pasaron a incorporarse como estímulos para la regeneración de áreas degradas, la atracción de nuevos habitantes o la potenciación turística de una ciudad (MONCLÚS \& GUARDIA 2006). Algunos autores señalaron que este aspecto se debía a la capacidad de las actividades culturales para atraer recursos públicos y privados a unos costes económicos y políticos limitados 
(STROM 2002). Este hecho implicó que en las últimas décadas prácticamente todas las ciudades occidentales hayan creado algún tipo de nuevo recurso cultural (LANDRY 2006). Incluso algunos autores apuntan que en buena medida, la posición estratégica de una ciudad entendida como un todo dependerá de los atractivos que sus instituciones culturales sean capaces de crear (FLORIDA 2010).

Siguiendo a MONTERO (2012: 79) esta aproximación implicaría repensar ciertos equipamientos culturales como una zona de contacto tal como postuló CLIFFORD (1999), basándose en las ideas y concepciones de la antropóloga PRATT (1992). Las zonas de contacto serían espacios de hibridación cultural donde se generarían varias tipologías de relaciones entre sujetos con posiciones dispares. Según esta visión, que se asemeja a la que ya proponía la Mesa redonda de Santiago de Chile en 1972 para el ámbito de los museos, la función del equipamiento cultural consiste en captar el máximo número de autores posibles para generar interrelaciones entre agentes sociales.

Los equipamientos culturales entran así en una lógica casi comercial de seducción. Se transforman en un elemento fundamental para definir la ciudad, para incorporarse en la competición mundial para atraer inversiones, visitantes $\mathrm{o}$ población $\mathrm{e}$ incluso, para el sentimiento de orgullo de sus ciudadanos (ZUKIN 1995). No obstante, este papel fundamental hace que las intervenciones aspiren a ser cada vez más ambiciosas y necesiten de inversiones mayores (CASTELLO 2010). Ahora bien, este incremento de las ambiciones no se ha acompañado de un incremento en la evaluación de las repercusiones. Así, la compleja relación entre aspectos como el apoyo a las industrias culturales, las mejoras urbanas, la ampliación de la educación de la población, el aumento del orgullo de los habitantes, el refuerzo de la cohesión social o la gestión de imagen turística supone una dispersión de los esfuerzos de los equipamientos culturales (SACCO 2006).

Conocer las repercusiones de la programación de un equipamiento resulta en consecuencia fundamental para conseguir los resultados previstos. El estudio pretende contribuir al debate actualmente en curso sobre las repercusiones de los equipamientos culturales en el entorno urbano. El actual modelo generalmente basado en la programación de eventos en espera de un público no definido se aleja del modelo óptimo. El estudio pretende mostrar posibles campos de mejora de las repercusiones de las distintas actividades en campos como la gestión de los riesgos, los costes de oportunidad o los beneficios de la programación.

Para ello se analizaran tres grandes áreas a las que varios estudios recientes (STYLIANOU-LAMBERT et al. 2014; AMBROSINO GUILLON 2013) consideran que se debe prestar una atención especial para establecer las relaciones entre los equipamientos culturales y la realidad social, cultural y económica de las áreas que los acogen: programación desarrollada, su proyección y los efectos inmateriales generados. En el apartado de metodología desarrollaremos los distintos indicadores utilizado para cada apartado.

El análisis de estas grandes áreas resulta representativo y complementario de las distintas tipologías de actividades que pueden desarrollar los equipamientos culturales. Desde actividades más hard, como la transformación física de un barrio, a actuaciones más soft, dirigidas por ejemplo a educar o fortalecer algún aspecto de la sociedad (GROTH \& CORIJN 2005). Así, la programación de un equipamiento puede aportar información relativa a la actividad desarrollada dentro de sus propias instalaciones (RUBIO AROSTEGUI \& RIUS ULLDEMOLINS 2012). La recopilación de información sobre la proyección de los equipamientos 
pretende analizar los esfuerzos realizados por este para hacer llegar sus actividades a un público amplio (PAÜL I AGUSTÍ 2014). Finalmente, el análisis de los efectos inmateriales generados se centrará en aquellas actuaciones de los distintos equipamientos focalizadas en responder a los retos de la sociedad; aspectos como la formación, el desarrollo económico o la creatividad en general (DEL BARRIO \& HERRERO 2014; LORENZEN \& FREDERIKSEN 2007).

\section{Los equipamientos culturales chilenos}

Los equipamientos culturales chilenos han experimentado un crecimiento importante en las últimas tres décadas. Este crecimiento ha sido aún más intenso como consecuencia de la inactividad en el campo cultural experimentada durante la dictadura militar, que paralizó prácticamente todos los proyectos en esta área (NAVARRO 2009). A partir de 1990 se inició una etapa de puesta al día y de creciente relevancia del rol de las administraciones públicas en materia cultural. Esta etapa aún está a la espera de un análisis crítico comparativo de las políticas desarrolladas (ANTOINE \& BRABLEC 2011; VEGA LAVANDEIRA \& ZEPEDA FARIAS 2010), por lo que únicamente podemos describir brevemente algunas de las actuaciones de cada período.

Durante el gobierno del Presidente Patricio Aylwin (1990 - 1994) se iniciaron varios proyectos culturales, centrados básicamente en la formación y en compensar los desequilibrios existentes. Muchos de estos proyectos se mantienen en la actualidad y se han transformado en equipamientos culturales reconocidos. Entre ellos podemos destacar espacios como la Corporación Cultural Balmaceda Arte Joven. Posteriormente el gobierno del presidente Eduardo Frei (1994 - 2000) realizó una tarea más legislativa, de debate y de creación de las bases para el desarrollo cultural. Se creó así una comisión asesora presidencial (comisión Ivelic) quien realizó un importante diagnóstico de las políticas culturales del país con el significativo título de "Chile está en deuda con la cultura". A nivel de infraestructuras se impulsó equipamientos como bibliotecas, teatros o museos (VEGA LAVANDEIRA \& ZEPEDA FARIAS 2010).

El gobierno del presidente Ricardo Lagos (2000 - 2006) a nivel de realizaciones destaca por algunos centros como Matucana 100, así como el impulso a un plan de mejora de varios museos los cuales, en muchos casos, no habían sido intervenidos de forma importante en 20 o 25 años (DIBAM 2002). Ahora bien, el aspecto más relevante fue la aprobación en 2003 de la creación del Consejo Nacional de la Cultura y las Artes, organismo encargado del desarrollo de las artes y la difusión de la cultura, dependiendo directamente de la presidencia de la República (ORTEGA RIQUELME 2012). Al final del mandato se aprobó otro documento con un título significativo "Chile quiere más cultura" para su desarrollo durante el período 2005-2010. Este plan aspiraba a actuaciones ambiciosas entre las que destacaba la decisión de dotar todas las comunas chilenas de más de 50.000 habitantes de una infraestructura cultural capaz de acoger representación de diferentes disciplinas artísticas (BRODSKY $\&$ NEGRÓN 2005). En total se trataba de 65 comunas que podían optar al financiamiento y asesoría del Plan (VEGA LAVANDEIRA \& ZEPEDA FARIAS 2010). Así mismo, se proponía capacitar a los profesionales locales para cumplir sus nuevas funciones culturales (FRUTOS \& MORENO 2008).

Se trataba de un plan ambicioso, desarrollado mayoritariamente por el gobierno de la presidenta Michelle Bachelet (2006 - 2010), en el que el presupuesto dedicado a la cultura se triplicó en valores absolutos (ANTOINE \& BRABLEC 2011) pese a seguir representando un valor relativamente bajo y estable alrededor del 0'4\% del presupuesto (OBSERVATORIO DE POLÍTICAS CULTURALES 2014). 
La continuidad de las políticas públicas en materia cultural de Chile es en este sentido remarcable (NAVARRO 2008).

Posteriormente, el gobierno del presidente Sebastián Piñera $(2010$ - 2014) seguiría en buena medida estas políticas, potenciando los contenidos de los equipamientos con programas como la red de teatros regionales, el de centros culturales o la reconstrucción patrimonial tras el fuerte terremoto de 2010. Una política seguida igualmente por su sucesora, Michelle Bachelet (2014, actualmente en el cargo), quien también ha iniciado una línea de fortalecimiento de los públicos y de las facilidades de acceso a la cultura (CONSEJO NACIONAL DE LA CULTURA Y LAS ARTES 2011; OBSERVATORIO DE POLÍTICA CULTURAL 2014). En conjunto, en la década 2003-2013 se han construido, rehabilitado o restaurado 157 espacios para las artes y la cultura de titularidad pública (ROJAS GOLDSACK 2014). Una cifra que muestra la ambición de la política desarrollada en estos últimos años. Pese a ello, algunos sectores señalan que la inversión realizada esconde aún una cuestión de fondo que no se ha abordado "la necesidad de pensar una política para el sector en su totalidad, una política participativa y congruente con las grandes transformaciones que la sociedad chilena requería para dejar, de una vez por todas, el espectro de la dictadura militar" (MATTHEY 2014: 19).

\section{MATERIALES Y MÉTODOS}

El artículo se basa en la comparación de equipamientos forzosamente distintos entre sí. Por ello se siguió parte de la metodología propuesta para el estudio comparativo de las políticas urbanas por KANTOR \& SAVITCH (2005). Se partió del estudio de la literatura existente, de visitas sobre el terreno y de entrevistas. Esta primera etapa ofreció una visión amplia de la problemática y permitió focalizarse posteriormente en la recopilación y estudio de aquellas variables más relevantes.

Concretamente, se trabajó con referencia a dos listados institucionales de equipamientos culturales. El primero de ellos fue Base Musa, con la que se identificaron un conjunto de 43 museos. Base Musa es un proyecto de la Subdirección Nacional de Museos, dependiente de la Dirección de Bibliotecas, Archivos y Museos (DIBAM), que pretende generar un catastro online de los museos que existen en Chile (http://www.basemusa. cl) (consultado 07/05/2014). Así mismo se analizó Red Cultura donde se identificaron un conjunto de 54 equipamientos suplementarios. Red Cultura pertenece al Consejo Nacional de la Cultura y las Artes y tiene como objetivo contribuir a un mejor acceso y participación de la población al arte y la cultura. (http://www.redcultura. cl/) (consultado 07/05/2014). El análisis de las dos bases sitúo el número inicial de equipamientos objeto de estudio en 97.

Entre los equipamientos que se analizaron encontramos espacios dedicados al arte (museos, teatros, salas de exposiciones) y espacios más transversales (bibliotecas, centros culturales). Con esta variedad se quería obtener una aproximación a lo que algunos autores denominan como hibridez de los espacios culturales (ORTEGA RIQUELME 2012), generando una visión lo más amplia posible.

Apartirdelos 97 equipamientosidentificados se hizo un primer vaciado de información a través de internet (webs, Facebook, blogs) En este proceso se descartaron algunos centros, básicamente aquellos con poca actividad y sin presencia propia en internet. Finalmente los centros objeto de estudio fueron 28. El listado de centros estudiados se puede consultar en la figura 4. Todos estos equipamientos fueron visitados, se recopiló información sobre su actividad a través de internet, material impreso y trabajos de investigación. Así mismo, se contactó con todos los centros para dar la 
oportunidad a los responsables de aportar información complementaria y contrastar los datos. Este último paso llevó a concretar 16 entrevistas a distintos responsables de equipamientos culturales. Se entrevistó responsables de (por orden de respuesta): Matucana 100, Estación Mapocho, Centro Cultural las Condes, Museo numismático Banco Chile, Casa Colorada - Museo de Santiago, Centro Cultural la Florida, Centro Cultural la Moneda, GAM (2 entrevistas), Centro Cultural Cerro Navia, Museo Interactivo Mirador, Balmaceda Arte Joven, MIL M2 (teatro Italia) y Museo Ralli. Las entrevistas fueron estructuradas, de unos 45 minutos, realizadas en castellano en los distintos centros. El rango de los entrevistados varió: directores, técnicos, responsables de aspectos concretos, etc. Las respuestas se recopilaron mediante notas. Agradecemos muy sinceramente la participación de los distintos entrevistados. Significativamente, en dos equipamientos estatales, el Museo Histórico Nacional y el Museo de Bellas Artes, se obtuvo respuesta positiva a través del Sistema Integral de Información y Atención Ciudadana (Ley $\left.\mathrm{N}^{\circ} 19.880\right)$ remitiendo a contactar por otro medio, sin finalmente conseguir fijar una fecha para la entrevista.

Durante las tareas de vaciado de la información y las entrevistas posteriores se buscó cuantificar información de tres grandes áreas analizadas: programación de los equipamientos culturales, proyección $\mathrm{y}$ efectos inmateriales generados. Concretamente se plantearon una serie de indicadores, todos ellos valorados con un punto y que sumaban un total de 10 para facilitar la comparación entre estas tres dimensiones.

La concreción de las distintas variables que conformaban el estudio se realizó siguiendo dos criterios prácticos. Por un lado, se buscaron aquellos indicadores más relevantes a partir del trabajo previo. Por otro lado, dada la variedad de equipamientos analizados, se buscaron indicadores que fácilmente estuviesen disponibles en todos los centros. Se descartó la elaboración o uso de indicadores complejos ya que muy difícilmente estaban disponibles en los diversos centros. Así mismo, debemos matizar que la información expresada, pese a estar cuantificada, no puede verse como una expresión inamovible de la situación de cada equipamiento. Dado que la programación tiende a ser cambiante, puede que un equipamiento salga sub o sobrerepresentado. En todo caso, la variedad de indicadores recopilados atenúa estos efectos y permite obtener una visión de conjunto de los equipamientos analizados.

Referentes a la primera variable analizada, la programación, se buscó un equilibrio entre las actividades desarrolladas por la institución, el análisis del público que asistía a los distintos equipamientos y la procedencia de los profesionales que llevaban a cabo las actividades (artistas en el teatro, conferenciantes, procedencia de las exposiciones). La elección de los criterios obedeció básicamente a la escasa disponibilidad de otros indicadores.

Los datos que finalmente se valoraron y los criterios de cuantificación se concretaron de la siguiente forma. Número de días de apertura (0'25 puntos por cada 90 días de apertura anual). Número de actividades (0'25 puntos por cada 5 actividades mensuales), su continuidad (0'25 puntos por cada 5 actividades desarrolladas durante más de un mes), la procedencia (0'25 puntos si el público era mayoritariamente local, 0'5 si metropolitano, 0'75 si estatal, 1 si era internacional), la edad ( 0 '25 puntos por cada componente de 15-25; 25-35; 35-60; más de 60 años); el nivel socioeconómico (0'25 puntos por cada componente $\mathrm{ABC} 1$, $\mathrm{C} 2 \mathrm{C} 3$, D o E) y la variabilidad de actores protagonistas de las actividades del centro (1 punto para cada componente si los actores participantes en las actividades del centro durante el último mes eran del barrio, metropolitanos, nacionales o internacionales). 
En cuanto al segundo aspecto, la proyección de los distintos centros culturales, se analizó a partir de dos variables ya utilizadas con éxito en trabajos anteriores (PAÜL I AGUSTÍ 2014): la propia voluntad del centro para darse a conocer y trabajar con en el entorno en el que se localiza (estudio de las campañas de difusión realizada por el centro y las colaboraciones existentes con otras entidades). Y los cambios que efectivamente hubiese podido generar estas actuaciones en el tejido urbano.

La cuantificación de los datos se concretó como sigue. Las campañas de difusión de los centros se valoraron con 4 puntos. Se otorgó hasta 1 punto a las campañas de cada uno de los ámbitos locales, metropolitanos, nacionales e internacionales. La puntuación de cada ámbito dependía de la duración y ambición de la presencia de las campañas promocionales. Para las colaboraciones efectivas entre instituciones se valoró la existencia de alguna actividad tangible, y se dió 0’25 puntos por cada entidad participante, hasta un máximo de 2 puntos. Los cambios en el transporte se valoraron con $0^{\prime} 25$ puntos por cambio enumerado desde la implantación de Transantiago (hasta un máximo de 1 punto). Los cambios en el sector turístico se valoraron a partir de la aparición de algún negoció dirigido a este público en un radio de 200 metros del centro (0’25 puntos por negocio, con un máximo de 1 punto). Los otros cambios comerciales se valoraron igualmente (con un máximo de 1 punto). Las transformaciones físicas se valoraron con 0'25 puntos (con un máximo de 1 punto) por cada actuación urbanística enumerada en relación directa con el centro cultural.

Finalmente, en lo que concierne a los efectos inmateriales generados se analizaron un conjunto de diez aspectos relacionados con los efectos generados por las "creatividades urbanas" (ZARLENGA et al. 2013), lo que recientemente se ha venido llamando en el contexto latinoamericano como Economía naranja (BUITRAGO RESTREPO \&
DUQUEMÁRQUEZ2013). Concretamente se analizaron datos referentes a la creación de barrios creativos, a los nuevos públicos, campañas, artistas o empresas generados, así como a la formación de las personas y la colaboración con el tejido industrial de la ciudad.

Los datos de este tercer aspecto se valoraron siempre sobre un máximo de 1 punto por epígrafe. Participación activa del centro en proyectos de barrios patrimoniales (0’25 puntos por cada ejemplo localizado). Nuevos públicos fidelizados (0'25 puntos por nuevo grupo de público asistente al equipamiento). Nuevas actividades artísticas programadas (0’25 puntos por nueva actividad desarrollada). Programación de compañías / artistas emergentes (0’25 puntos por ejemplo). Nuevas empresas surgidas del trabajo del equipamiento (0'25 puntos por ejemplo). Nuevos artistas surgidos de la programación del equipamiento (0'25 puntos por ejemplo). Productos desarrollados y comercializados por el centro (0'25 puntos por ejemplo). Colaboración con empresas ya existentes (0’25 puntos por ejemplo). Colaboración con otras instituciones culturales (0'25 puntos por ejemplo efectivo de colaboración). Tareas de formación (0’25 puntos por curso desarrollado).

\section{RESULTADOS}

\section{Valoración de la programación de los equipamientos culturales}

La programación de los equipamientos culturales tiende a actuar como su carta de presentación. En este sentido, su análisis puede aportar una valiosa información sobre los aspectos que prioriza el propio equipamiento cultural en su misión. Según los datos obtenidos, recopilados en la figura 1, la programación de los centros culturales de Santiago de Chile se caracterizaría por una priorización de la oferta. Así, los distintos centros abren sus puertas de forma 
amplia, realizan un número de actividades elevado y tienen cierta continuidad en sus actividades. Ahora bien, en términos generales, a partir del material recopilado, no se ha observado una correlación entre estos aspectos y la voluntad efectiva de llegar a un público amplio. A modo de ejemplo, la fijación de los días de apertura de los distintos centros tiende a basarse más en criterios administrativos (básicamente disponibilidad de personal), que en una reflexión sobre los días que más le convendrían al posible visitante.

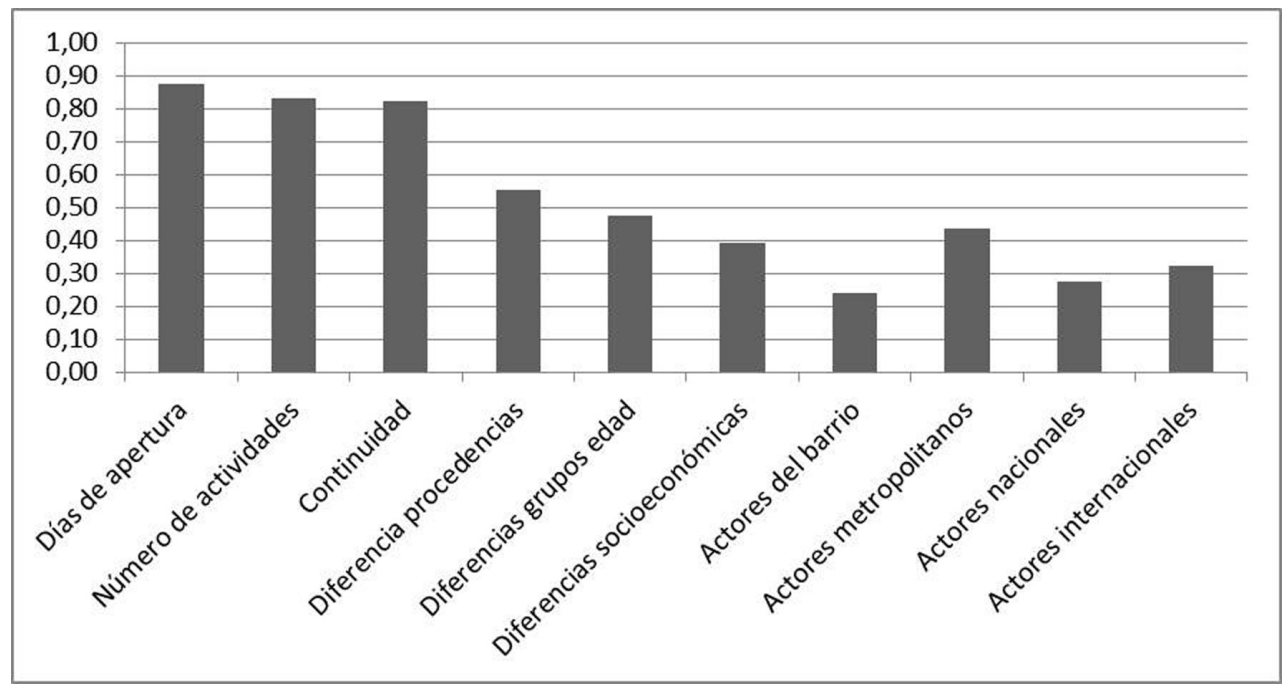

Figura 1. Resumen de resultados relativos a la programación de los equipamientos culturales.

Figure 1. Summary of results relating to cultural programming aspects on the cultural facilities.

No obstante, esta amplitud en términos de programación de actividades no siempre repercute en el conjunto de la población. Como se observa, los datos relativos a procedencia de visitantes, grupos de edad y especialmente variedad de nivel socioeconómico de los visitantes son sensiblemente más bajos. La simple apertura de los distintos centros no consigue que el conjunto de la población los visite. Una situación que limita las repercusiones de los propios centros, ya que sus actividades únicamente benefician a una parte de la población.

Esta problemática, habitual en la mayoría de equipamientos culturales, puede ser combatida con políticas activas de captación de públicos. A lo largo del estudio hemos encontrado algunas experiencias interesantes en esta línea, por ejemplo el proyecto del Museo Interactivo Mirador "MIM verano vecino" con la población de los alrededores o las facilidades que el Museo Numismático del Banco Central de Chile pone para el desplazamiento al museo de alumnos de escuelas de ciertos barrios. Ahora bien, en la mayoría de casos se trataba de actuaciones dirigidas a escolares. Las políticas dirigidas a otros colectivos son prácticamente inexistentes, lo que limita la difusión de los beneficios al conjunto de la sociedad.

Por último, en términos de procedencia de los distintos actores que realizan las actividades puntuales en los centros, encontramos nuevamente unas limitaciones importantes. En primer lugar debemos matizar que en 10 de los equipamientos 
analizados el volumen de actividad era escaso o nulo. Centros como el Museo Postal y Telegráfico o el Museo San Francisco basan su actividad de forma casi exclusiva en su colección permanente, lo que limita las posibles repercusiones de su programación.

En los centros con programación la mayoría de los actores procedían del área metropolitana de Santiago. En muy pocos casos los actores residían en el propio barrio. De hecho, en términos generales, sorprende que incluso haya mayor presencia de artistas internacionales que locales. Así, únicamente el Centro Cultural de Cerro Navia disponía de una programación militantemente local. Una situación sobre la que volveremos más adelante, y que puede tener una lectura positiva, en acercar a la población local actividades creadas en otros ámbitos, pero que también puede dificultar la promoción del tejido cultural del propio barrio. Además, en el contexto de Santiago, donde como hemos visto la mayoría de asistentes a los equipamientos pertenecen a unas clases definidas, acomodadas, nos inclinamos por valorar este hecho como una limitación a la creación y promoción de las compañías del propio barrio. Unos hechos que limitan el enriquecimiento del tejido local y la difusión de políticas colaborativas en estos contextos.

\section{Proyección de la actividad de los equipamientos culturales}

La segunda de las variables analizadas, la relativa a las actividades que los equipamientos culturales desarrollan fuera de su sede física, la encontramos recopilada en la figura 2. En términos generales se puede observar unos valores claramente inferiores a los del apartado anterior. Con la excepción de las campañas de difusión (prensa, anuncios, presencia en internet, etc.) la mayoría de valores observados son bajos. Incluso en muchos de los centros los aspectos analizados son inexistentes.

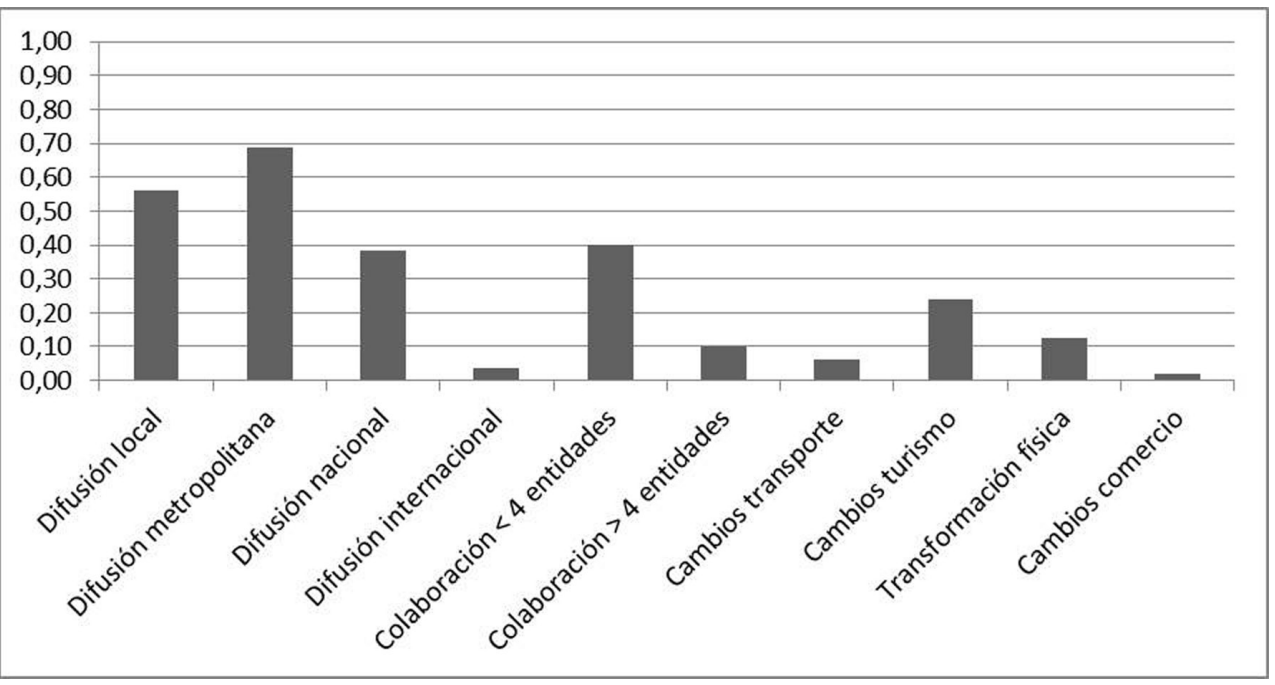

Figura 2. Resumen de resultados relativos a la proyección de los equipamientos culturales.

Figure 2. Summary of results for the dissemination aspects on the cultural facilities. 
En términos de comunicación existe una preocupación evidente en la mayoría de centros por difundir sus actividades. La concienciación en este sentido es elevada, pese a que las limitaciones de presupuesto y personal hacen que las campañas no siempre sean las deseadas. No nos extenderemos en este punto, ampliamente tratado por varios autores (MIRONER 2001). Únicamente apuntaremos algunas limitaciones observadas que retroalimentan algunos de los aspectos descritos en el apartado anterior. Así, resulta sorprendente que se observe unos valores más elevados en la comunicación de ámbito metropolitano que en la propia comuna en la que se implantan los equipamientos ${ }^{2}$. En lo que concierne la difusión nacional e internacional, esta tiende a realizarse básicamente por los centros nacionales.

Muchos centros dirigen su comunicación al conjunto del área metropolitana de Santiago, con el objetivo de conseguir un aumento genérico de audiencias, sin ningún tipo de atención especial a los vecinos del equipamiento. Se prioriza, así, la difusión de la actividad a la apuesta por la difusión de los contenidos entre los ámbitos más cercanos. En este sentido, se establece una relación basada en los eventos más que una fidelización de los visitantes y una posibilidad de trabajo a largo plazo. Es más, en los pocos casos en los que se busca la interrelación con el vecino se acostumbra a fijar a través del precio. Este es el caso del Centro Cultural las Condes, que promociona los descuentos que reciben los vecinos en el precio de las actividades. Una notable excepción a esta situación es la del GAM. Este centro desarrolla una estrategia para interactuar con los vecinos (tarjeta específica para los vecinos, desarrollo de

2 En muchos casos la comunicación metropolitana incluye también la propia comuna de implantación del equipamiento. Pese a ello, se trata de una comunicación generalizada, sin atender a peculiaridades locales. actividades conjuntas, intercambio de ideas, etc.), con unos buenos resultados para el desarrollo del barrio de Lastarria.

Las limitadas relaciones con los vecinos se reflejan también en la apertura de los centros a las colaboraciones con otras entidades. En once de los centros estudiados no hemos encontrado ninguna colaboración con entidades externas ${ }^{3}$. En el otro extremo, únicamente cuatro centros tenían cinco o más relaciones y de ellos únicamente en dos casos las interrelaciones se establecían con entidades próximas ${ }^{4}$. Un panorama limitado, que muestra unas instituciones culturales aún cerradas en ellas mismas y sin interrelaciones con el tejido económico o social de la ciudad.

El aspecto anterior se ve aún más reforzado si analizamos las repercusiones que los centros culturales generan en sus alrededores. En términos generales se admite que estos espacios pueden contribuir a impulsar transformaciones físicas relevantes en los barrios en los que se implantan. Ahora bien, en el análisis desarrollado se ha podido comprobar que los cambios a nivel de transporte, turismo, urbanismo o comercio que los distintos centros chilenos enumeran como repercusiones son extremadamente limitados.

3 Se entendía por colaboración aquella relación en la que existía un intercambio de experiencias entre el centro cultural y la empresa, agrupación o institución externa. Se han excluido aquellos intercambios únicamente de carácter económico por prestación de obra o servicio.

4 Se trataba del GAM y Balmaceda Arte Joven con relaciones básicamente con el tejido cultural y artístico del barrio. Los otros dos centros tenían relaciones más especializadas. El Centro Cultural la Moneda mantiene contactos regulares con instituciones similares a escala internacional y el Museo Nacional de Historia Natural Chile hace lo mismo con instituciones científicas de todo el mundo. 
Así únicamente el Museo de la Memoria y de los Derechos Humanos generó una repercusión destacada en términos de transporte (un nuevo acceso al metro). Los cambios en el sector turístico tienden a ser igualmente limitados. A modo de ejemplo, únicamente fueron citados como relevantes por los responsables de Estación Mapocho, el Centro Cultural la Moneda y el GAM (que incluyó una oficina de información turística en sus instalaciones). A nivel urbanístico nuevamente fueron estas tres instituciones las que relataron cambios importantes en los alrededores impulsados por su presencia (generalmente habilitación de nuevas plazas en las inmediaciones del centro). En términos comerciales únicamente se recopilaron cambios relevantes alrededor del GAM y del Teatro Italia.
Esta limitación de las repercusiones de los centros en el tejido físico de la ciudad es una diferencia fundamental con los museos europeos (PAÜL I AGUSTÍ 2014), lo que limita el efecto multiplicador del centro y los potenciales beneficios para el conjunto de la ciudad.

\section{Efectos inmateriales de la actividad de los equipamientos culturales.}

Elúltimo grupo de repercusiones potenciales analizadas fueron los efectos inmateriales de la actividad de los equipamientos, recopilado en la figura 3. Estos datos muestran aquellos impactos sin efectos físicos directos, pero que pueden contribuir al fortalecimiento del tejido económico, social y cultural de las distintas ciudades.

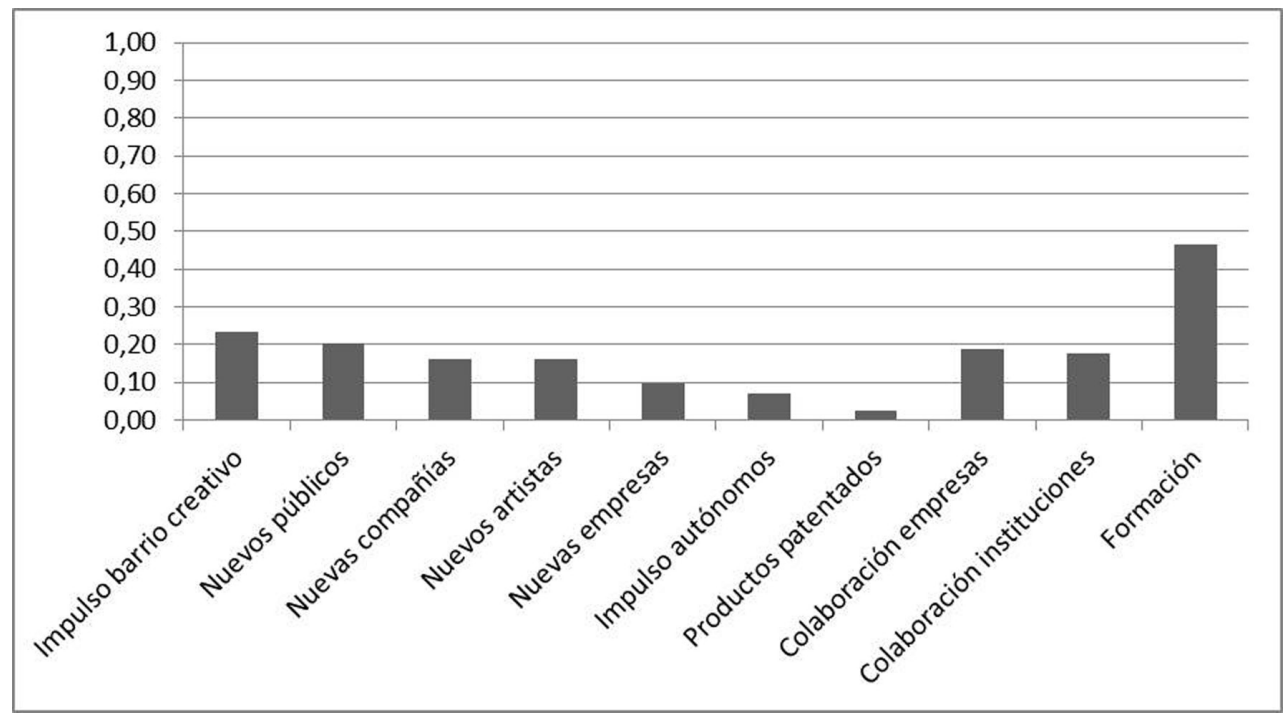

Figura 3. Resumen de resultados relativos a los efectos inmateriales de los equipamientos culturales.

Figure 3. Summary of results relating to intangible effects on the cultural facilities.

Los datos recopilados muestran los escasos valores que consiguen los equipamientos analizados en Santiago de Chile. La mayoría de centros no tienen ningún tipo de política desarrollada en este campo. Únicamente la formación, básicamente entendida como actividades dirigidas al público escolar, consigue sobresalir sin ser, no obstante, mayoritaria en todos los centros. El papel de los centros culturales como transmisores 
de conocimiento queda, de este modo, limitado.

Lo mismo se puede comentar del impulso a los barrios creativos, entendido como aquellas actuaciones dirigidas a potenciar, en un sentido amplio, la formación, difusión o participación de la ciudadanía en dinámicas sociales positivas para el área (RIUS ULLDEMOLINS \& ZARLENGA 2014). En la mayoría de centros no existe ninguna política conjunta dirigida a fomentar este aspecto. Únicamente unos pocos centros, como el GAM, Balmaceda Arte Joven o Matucana 100 trabajan en esta línea, completamente desconocida por la mayoría de centros analizados.

La misma problemática se repite a nivel más de detalle. La incorporación efectiva de nuevos públicos ${ }^{5}$ pese a estar recogida como misión propia de distintos centros, tiende a carecer de estrategias para su implementación efectiva y únicamente hemos contabilizado algunas experiencias puntuales.

La creación de nuevas compañías artísticas o el apoyo a los artistas emergentes tampoco es prioritaria para la mayoría de espacios, con la notable excepción de Balmaceda Arte Joven o teatro Italia. La mayoría de centros consideran que esta no es su misión y apuntan que sería responsabilidad de los Fondos de Cultura del Consejo Nacional de la Cultura y las Artes. En varios centros incluso se señaló que únicamente se programaba artistas consagrados, viendo la consagración como sinónimo de calidad. Un aspecto que podría dificultar la creación de un tejido cultural en Santiago de Chile, pero que también puede contribuir a crear una escena cultural alternativa.

Se entiende por incorporación de nuevos públicos la apertura a nuevos colectivos, no el aumento de las visitas de colectivos ya presentes en las actividades de las distintas instituciones.
El impulso a nuevas empresas culturales (gestión, iluminación, traslado de obras, etc.) también tiende a ser escaso. Únicamente Matucana 100 y Balmaceda Arte Joven apuntaron como punto fuerte de su gestión la consolidación de empresas privadas capaces de generar productos culturales independientes del centro. Lo mismo podemos apuntar de la creación de nuevos productos comercializables. Un aspecto que sólo se comentó en el GAM (experiencia Residencia Riolab) y Balmaceda Arte Joven (con aspectos de gestión de salas). Únicamente las colaboraciones con empresas e instituciones, mediante intercambio económico, parecen sobresalir. Ahora bien, se trata generalmente de compras sin capacidad de influir en la empresa o bien de subvenciones recibidas por parte de administraciones públicas. La apuesta por este conjunto de aspectos podría contribuir a generar cierto efecto multiplicador. Ahora bien, en los términos actuales todo parece indicar que este efecto es, en el caso de Santiago de Chile, limitado.

\section{La interacción de los efectos en los museos de Santiago de Chile.}

En los apartados anteriores hemos ofrecido una visión conjunta y por ámbitos de las distintas variables incorporadas en el estudio. No obstante, las diferencias entre los centros analizados son evidentes. Así, en la figura 4, hemos incorporado los resultados individualizados de cada uno de los centros culturales. 


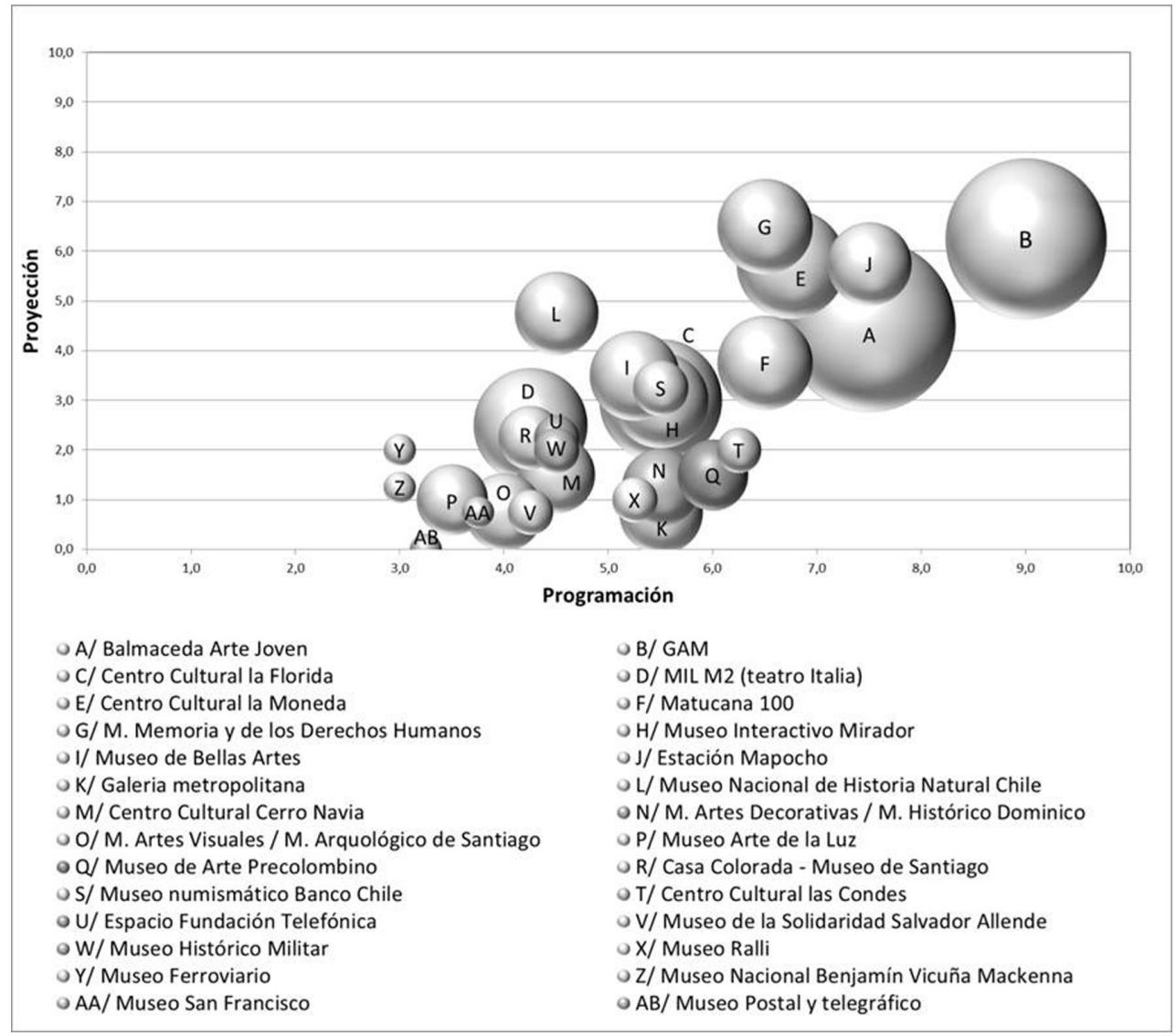

Figura 4. Impacto de los museos según programación (eje $x$ ), proyección (eje y) y efectos inmateriales (tamaño). Fuente: Para el valor de los efectos inmateriales, referente al tamaño, oscila entre un valor 0,25 para el Museo Ferroviario (Y); 1,75 para el Museo Nacional de Historia Natural Chile (L) y 7,5 para Balmaceda Arte Joven (A).

Figure 4. Effects of museums according to cultural programming ( $x$ axis), dissemination (y axis) and intangible aspects (size). Source: For the value of immaterial effects, about size ranging from 0.25 to The Railway Museum (Y); 1.75 to The Chilean National Museum of Natural History (L) and 7.5 to Balmaceda Arte Joven (A).

Como se puede observar, los datos obtenidos parecen indicar ciertas interrelaciones entre las tres variables analizadas. En términos generales la programación de los distintos centros tiende a ser su ámbito más fuerte y dónde la mayoría obtienen mejores resultados. Además, la proyección de estos centros se incrementaría cuando se consigue cierto nivel de programación. Por último, los efectos inmateriales en el territorio tienden a ser relevantes cuando se obtiene cierto nivel de programación y de difusión.

En los niveles más bajos de programación encontramos museos extremadamente diversos a nivel temático, de programación y de propiedad. Centros como el Museo Ferroviario, el Museo Nacional Benjamín Vicuña Mackenna o el Museo Postal y Telegráfico. En general, no obstante, tiende 
a tratarse de equipamientos relativamente modestos en infraestructura, con tradición $\mathrm{y} \sin$ un equipo amplio que lo gestione. En cambio en el otro extremo, los centros con mayor actividad, coinciden con los que tienen mayores recursos económicos. En este sentido es significativo que entre los seis centros con mayor actividad aparezcan los cuatro centros analizados que reciben transferencias directas del Consejo Nacional de la Cultura y las Artes $^{6}$ (OBSERVATORIO DE POLÍTICA CULTURAL 2014). Además encontramos el Museo de la Memoria y de los Derechos Humanos, con aportes importantes de fondos gubernamentales. Únicamente encontraríamos Estación Mapocho como centro autofinanciado sin transferencias de fondos públicos. La relación entre recursos económicos disponibles y capacidad de programación parece en este punto evidente. Una relación, la anterior, que se acentúa en los siguientes puntos analizados.

La distribución de las instituciones descrita para la programación también es válida para la promoción. En la mayoría de casos los resultados de los distintos centros se sitúan en puntos comparables. Únicamente encontramos posiciones relativamente distintas en algunos casos puntuales. Este es el caso del mayor acento a la promoción del Centro Cultural Palacio de la Moneda, situación que podríamos atribuir a su posicionamiento en el distrito cívico de la ciudad, alejado del grueso de la población, así a como su arquitectura subterránea. Ambos factores le obligarían a una mayor promoción para darse a conocer. En el otro extremo, el Museo de Artes Decorativas / Museo Histórico Dominico realizaría más actividades de las que proyecta.

La mayor diferencia detectada entre programación y promoción vendría, no obstante, de las intensidades claramente

6 Por orden de mayor a menor nivel de actividad: GAM, Balmaceda Arte Joven, Centro Cultural la Moneda y Matucana 100. diferentes de ambas. Mientras que en la programación los cinco centros con valores más altos multiplican por dos el resultado de los cinco centros con valores más bajos, en el campo de la promoción los valores se multiplican por seis. De estos resultados se puede extraer una doble lectura. En primer lugar positiva. Se considera que la programación de los distintos equipamientos se ve como una prioridad y como la propia razón de la existencia de los centros. La idea que sin programación el centro no tendría motivo para existir se encuentra ampliamente extendida entre los centros chilenos, lo que genera un volumen de actividad notable. Ahora bien, existiría una segundo lectura, más en clave de deficiencias. Se programa mucho, pero ¿el público sabe lo que se programa? Según los datos disponibles, la difusión amplia de la programación más allá de la publicidad dirigida a los círculos habituales es limitada. Un aspecto que muchos técnicos entrevistados relacionan con limitaciones presupuestarias, pero que debería ser objeto de una mayor reflexión.

Sin una buena difusión entre el público potencial, las repercusiones de los distintos equipamientos culturales sobre su entrono tiende a ser menores. Al mismo tiempo surgen dudas sobre los efectos que las distintas actividades pueden generar en su entorno. A modo de ejemplo podemos citar el Centro Cultural las Condes, con unos indicadores en términos de programación superiores a la media, pero con una difusión limitada. ¿El conjunto de la población conoce la oferta cultural desarrollada? ¿Se planifica una difusión para todos los segmentos sociales? ¿Se busca interrelacionar el centro con el tejido social, económico o educativo de la ciudad? A la luz de los resultados obtenidos parece que en un buen número de centros estas no son las prioridades.

Finalmente, en lo que concierne a la tercera de las variables, los efectos inmateriales, las diferencias son aún más acentuadas. Las 
diferencias entre los valores obtenidos en los cinco equipamientos con mejor y con menor puntuación es de 19 veces. Es más, en la mayoría de centros las políticas de creación de efectos inmateriales en el entorno urbano son prácticamente inexistentes o un efecto en buena medida imprevisto de las actuaciones de las distintas instituciones. Esto contrasta con la concienciación existente sobre la temática en el discurso oficial de los distintos equipamientos analizados, donde las interrelaciones con la comunidad están muy presentes ${ }^{7}$. Una situación que se aleja de los datos recopilados que muestran una escasa atención a lo que sucede fuera del recinto de las instituciones.

Pese a esta situación general existen algunos centros con una activa voluntad de crear sinergias con el entorno social. Se trata especialmente del GAM y de Balmaceda Arte Joven. En ambos casos se ha podido observar una clara voluntad de los respectivos centros para interrelacionarse con el entorno. En el caso del GAM con iniciativas como el trabajo desarrollado con el tejido asociativo del barrio de Lastarria, comentado anteriormente, pero también con proyectos como Barrio Escuela. Un proyecto dirigido a fomentar la participación cultural de jóvenes entre 15 a 25 años residentes en comunas alejadas del centro de Santiago. O el trabajo de intermediación $\mathrm{y}$ acercamiento a comunidades que usan los espacios exteriores del GAM para sus

7 A modo de ejemplo podemos citar la Visión del Museo de Arte Colonial de San Francisco que "aspira a ser una institución de referencia, que comunique y refleje la época colonial americana, a través de un diálogo plural con la comunidad en todas sus expresiones" http://museosanfrancisco.com/ mision-y-vision/ (consultada el 12/04/2015). O la del Museo Histórico y Militar "Un museo de excelencia (...), que entregue cultura de calidad, manteniendo un diálogo permanente con la comunidad nacional e internacional" https://www.youtube.com/ watch? $\mathrm{v}=\mathrm{X}$ 9mGQU7rGGM (consultada el 12/04/2015). actividades (A-Pop, patinadores o baile latino). Un público que no participaba de las actividades del centro y con los que el GAM trabaja activamente para atraer y compatibilizar sus actividades con la programación del centro. En el caso de Balmaceda Arte Joven las interrelaciones con el entorno también resultan evidentes y una de las misiones del centro (MELLA 2008). Su propia actividad se dirige a la formación e interacción entre artistas, con una programación que ha evolucionado con los años para responder a los cambios de la sociedad.

Algunos otros pocos centros también desarrollan actividades en esta línea, pese a que tienden a ser más puntuales. A modo de ejemplo podemos citar el Centro Cultural de la Florida, que desarrolló un catálogo de artistas presentes en el barrio para potenciar sus interrelaciones. De Teatro Italia, con una programación abierta a las experiencias y propuestas del barrio. O por último, Matucana 100 y su experiencia de colección vecinal, instalación realizada con el apoyo de los vecinos del área. No obstante, podemos considerar que actualmente la programación y la difusión de las actuaciones de los distintos equipamientos tienden a formar parte de las políticas de los centros culturales de Santiago de Chile, mientras que las interrelaciones con el tejido social son una asignatura pendiente que limita las potenciales repercusiones sociales, económicas e incluso culturales de los distintos equipamientos culturales.

\section{LOS EQUIPAMIENTOS CULTURALES DE SANTIAGO DE CHILE: UNOS CENTROS CON POCAS INTERRELACIONES CON LOS BARRIOS}

Como se ha analizado, las actividades de los equipamientos culturales de Santiago de Chile tienden a focalizarse en las distintas actividades que desarrollan dentro de su propio equipamiento y a 
algunas actividades, menos habituales, de difusión entre la ciudadanía. La colaboración con el tejido social tiende a ser extremadamente limitada y seguir una aproximación top-down en la que el papel del conjunto de la sociedad queda sujeto a los deseos del centro. Salvo en casos puntuales las distintas instituciones parecen priorizar su propia programación e ignorar las posibles colaboraciones, actividades y expectativas de la población a la que dirigen sus actividades. En este sentido existe una cierta tendencia a "programar por programar", siguiendo los propios criterios del centro, y esperar a que la propia calidad del producto, que no analizamos, unida a algunas actuaciones puntuales (especialmente la gratuidad puntual), sea suficiente para formar nuevas audiencias y contribuir a generar repercusiones positivas en la sociedad. Una situación que dista de ser la óptima, pero que está experimentando algunos cambios.

A lo largo de la investigación hemos podido comprobar como en varios de los responsables de los equipamientos existe una voluntad de ampliar sus actividades hacia la sociedad. Se trata, en términos generales, de nuevos técnicos formados e incorporados a los distintos equipamientos en los últimos años. Un hecho que poco a poco está cambiando las mentalidades y las metodologías de trabajo ya que como alertaba MATTHEY (2013: 10) "así y todo en Chile aún falta profesionalizar la gestión cultural". Igualmente, el trabajo realizado por algunos centros de referencia, especialmente el GAM, conocido y generalmente citado como elemento a seguir, empieza a cambiar algunas rutinas entre los técnicos. Unos técnicos, no obstante, que se encuentran con algunas limitaciones materiales importantes.

Así, en términos generales, en la última década los centros culturales chilenos se han ampliado enormemente tanto en número, importancia como ambición. Como recopilaba ARTURO NAVARRO (2008: 45) "el desafío es inmenso y apasionante. Estamos a las puertas de dar un salto cualitativo en la gestión cultural chilena". Ahora bien, en buena medida podríamos aplicar a los museos y centros culturales analizados el mismo diagnóstico que ya realizaba ROJAS GOLDSACK (2014: 11) para el caso de los teatros: "Sin duda no es suficiente contar con más y mejores espacios para la cultura a lo largo del territorio nacional (...) a su vez requerimos una ciudadanía que participe en el proceso de instalación de estos espacios como polos de desarrollo social y cultural de la propia comunidad".

Este salto hacia la ciudadanía supone una apuesta importante. La inversión realizada en las últimas décadas ha sido importante, pero dirigida especialmente hacia la infraestructura física. El reto para los próximos años debería ser la gestión de los equipamientos ya creados para fomentar interrelaciones lo más beneficiosas posibles con el conjunto de la población. En este sentido, a partir de los datos obtenidos, apuntamos a modo de conclusiones algunas debilidades que los equipamientos culturales de Santiago de Chile deberían plantearse para mejorar su integración en el tejido urbano y sus interrelaciones con la sociedad:

- Resulta sorprendente la dicotomía existente entre equipamientos culturales locales y estatales observada en las entrevistas y apuntada también por la Ministra Presidenta del Consejo Nacional de la Cultura y las Artes (URRUTIA FERNÁNDEZ 2008: 20). Con el modelo actual muy difícilmente la población local interactuará con la programación de los equipamientos de proyección estatal. Del mismo modo, los equipamientos locales quedaran restringidos a actividades de proximidad, sin interacción con otros ámbitos. Una mayor hibridación de la programación, como la apuntada para 
los Centros Culturales Comunales según una clasificación "en espacios de proximidad y/o de centralidad, según su ámbito de acción, sea éste demográfico o geográfico" (MORALES ARIAS 2011: 15) enriquecería el equipamiento, la población y los artistas participante.

- Una mayor interrelación entre instituciones públicas contribuiría a mejorar la proyección de los distintos equipamientos. Actualmente los equipamientos tienden a impulsarse en solitario por la entidad responsable (FARÍAS 2004). Una colaboración más estrecha con otras unidades, como el transporte, vía pública, jardines o limpieza ayudaría a mejorar las repercusiones generadas y evitaría algunas situaciones como centros sin transporte público o equipamientos en calles con una urbanización claramente deficiente.

- En términos generales deberían reforzarse las plantillas de trabajadores de la mayoría de instituciones. Como recopilan algunos autores "los municipios, en este momento, cuentan con un nivel de gestión cultural muy precario" (VEGA LAVANDEIRA \& ZEPEDA FARIAS 2010: 87). En los equipamientos de otra titularidad la situación no parece mejor y a menudo se sustenta por el empeño y la juventud del equipo. Ante el salto cualitativo que están experimentando los equipamientos culturales chilenos estos últimos años se impone una inversión en capital humano y en modernizar y agilizar las estructuras de gestión. De lo contrario muy difícilmente se podrán asumir los distintos objetivos que se plantean los nuevos equipamientos y se correrá el riesgo de limitar sus repercusiones a los públicos y contextos limitados que habitualmente ya se benefician de las actuaciones culturales.

\section{AGRADECIMIENTOS}

El artículo se integra dentro del proyecto financiado por el Plan Nacional de $\mathrm{I}+\mathrm{D}+\mathrm{i}$ «Competitividad e innovación en el desarrollo territorial: definiendo las bases de un nuevo modelo socio-económico para una España post-crisis» (CSO201239373-C04-02). También ha sido posible gracias a la financiación del Grup de Recerca Consolidat «Territori i Societat» de la Generalitat de Catalunya (2014 SGR 973). La estancia sobre el terreno fue financiada por las becas Iberoamérica para Jóvenes Profesores Investigadores 2014 del Banco Santander. Se agradece así mismo la colaboración del profesor Dr. Jorge Ortiz durante la estancia en la Universidad de Chile.

\section{BIBLIOGRAFÍA}

AMBROSINO, C. \& V. GUILLON, 2013. La metropole des arts creatif. Technoscience et culture du libre. Programme de Recherches Territorialisées en RhôneAlpes, Lyon.

ANTOINE, C. \& D. BRABLEC, 2011. Políticas Culturales: La Acción del Estado y la Sociedad de Oportunidades. Libertad y Desarrollo, Santiago de Chile.

BARKE, M.\& K.HARROP, 1994. Selling thee industrial town: identity, image and illusion. In: GOLD, J. y S. WARD (Eds.), Place, promotion: the use of publicity and marketing to sell towns and regions. Wiley, Chichester: 93-113.

BIANCHINI, F. \& M. PARKINSON (Eds.), 1993. Cultural policy and urban regeneration: the West European experience. Manchester University Press, Manchester. 
BRODSKY, R. \& B. NEGRÓN, 2005. Chile quiere más cultura. Definición de política cultural 2005-2010. Consejo Nacional de Cultura y las Artes, Valparaíso. http://www.cultura.gob.cl/wpcontent/uploads/2012/03/Chile-QuiereM\%C3\%A1s-Cultura.-Definiciones-dePol\%C3\%ADtica-Cultural-2005-2010.pdf

BUITRAGO RESTREPO, F. \& I. DUQUE MÁRQUEZ, 2013. La economía naranja. Una oportunidad infinita. Banco Interamericano de Desarrollo / Puntoaparte, Washington / Bogotá.

CASTELLO, L., 2010. Rethinking the meaning of place: conceiving place in architecture-urbanism. Ashgate, Farnham.

CLIFFORD， J., 1999. Los Museos como Zonas de Contacto. In: Clifford, J. (Ed.) Itinerarios Transculturales. Gesida: Barcelona: 233-271.

CONSEJO NACIONAL DE LA CULTURA Y LAS ARTES, 2011. Política cultural 2011-2016. Consejo Nacional de la Cultura y las Artes, Valparaíso.

DE GRAAFF, T., J. BOTER \& J. ROUWENDAL, 2009. On spatial differences in the attractiveness of Dutch museums. Environment and planning A, 41 (11): 2778-2797.

DEL BARRIO, M. J. \& L. C. HERRERO, 2014. Evaluating the efficiency of museus using múltiple poutputs: evidence from a regional System of museus in Spain. Intwernational journal of cultural Policy 20 (2): 221-238.

\section{DIRECCIÓN DE BIBLIOTECAS} ARCHIVOS Y MUSEOS (DIBAM), 2002. Plan Nacional de Mejoramiento Integral de los Museos. http://www.dibam.cl

EVANS, G., 2001. Cultural planning an urban renaissance? Routledge, London.
EVANS, G. \& J. FOORD, 2008. Cultural mapping and sustainable communities: planning for the arts revisited. Cultural trends, 17 (2): 65-96.

FARÍAS CABALLERO, E., 2014. Estudio nacional de gestión cultural municipal Observatorio cultural, http:// www.observatoriocultural.gob.cl/revista/7estudios/22-estudio-nacional-de-gestioncultural-municipal/

FLORIDA, R., 2010. The great reset: how new ways of living and working drive postcrash prosperity. Harper, New York.

FRUTOS, M. \& J. MORENO, 2008. Programa de Centros Culturales CNCA. In: García, F. (Ed). Centros Culturales: Proyecciones, Infraestructura y gestión. Consejo Nacional de la Cultura y las Artes, Valparaíso.

GARCÍA, B., 2004. Urban regeneration, arts programming and major events: Glasgow 1990, Sydney 2000 and Barcelona 2004. International journal of cultural policy, 10 (1): 103-118.

GROTH, J. \& E. CORIJN, 2005. Reclaiming Urbanity: Indeterminate Spaces, Informal Actors and Urban Agenda Setting. Urban Studies, 42, (3): 503-526.

KANTOR, P. \& H. V. SAVITCH, 2005. How to Study Comparative Urban Development Politics: A Research Note. International Jornal of Urban and Regional Research, 291: 135-151.

KEMP, R.L. (Ed.), 2004. Cities and the arts. A handbook for renewal. McFarland and Company, London.

LANDRY, C., 2006. Culture and Régéneration Urbaine. Approche intégrée. Le rôle de la culture et de la créativité dans le (re)développement des villes. UrbactLille Métropole, Lille. 
LORENZEN M. \& L. FREDERIKSEN, 2007. Why do cultural industries cluster? Localization, urbanization, products and projects. In: Cooke, P. Y R. Lazzeretti (Eds.). Creative Cities, Cultural Clusters and Local Economic Development. Edward Elgar Publishing, Cheltenham: 155-179.

MATTHEY, G., 2013. La gestión cultural en Chile, a pasos de su "segunda generación". MGC Revista Gestión Cultural, 1: 6-11.

MATTHEY, G., 2014. ¿Gestión de las artes en Chile?: la urgencia de una cuestión previa. MGC Revista Gestión Cultural, 3: 18-20.

MELLA, F., 2008. Experiencia de gestión en Balmaceda 1215. In: Consejo Nacional de la Cultura y las Artes, Centros Culturales. Proyección, infraestructura y gestión. Consejo Nacional de la Cultura y las Artes, Valparaíso.

MIRONER, L., 2001. Cent musées à la rencontre du public. France Edition, Cabestany.

MONCLÚS, J. \& M. GUÀRDIA (Eds.), 2006. Culture, urbanism and planning. Ashgate, Aldershot.

MORALES ARIAS, P. (Coord.), 2011. Guía Introducción a la gestión e infraestructura de un Centro Cultural comunal. Consejo Nacional de la Cultura y las Artes, Valparaíso.

NAVARRO, A., 2008. Renaciendo desde los sueños, la ocupación y las cenizas. In: Consejo Nacional de la Cultura y las Artes, Centros Culturales. Proyección, infraestructura y gestión. Consejo Nacional de la Cultura y las Artes: Valparaíso.

NAVARRO, A., 2009. Museos y centros culturales. http://arturo-navarro.blogspot. be/2009/11/una-falsa-disyuntiva-y-lapiramide-de.html
NAVARRETE, D., 2008. Nouveaux musées dans la région parisiense. Téoros, 27 (3): 71-77.

\section{OBSERVATORIO DE POLÍTICA} CULTURAL, 2014. Informe Situación Presupuestaria en Cultura 2014. http://www. observatoriopoliticasculturales.cl/OPC/ seguimiento/situacion-presupuestaria-2014/

ORTEGA RIQUELME, F., 2012. Redes culturales. Un intercambio internacional para la descentralizacion del arte y la equidad cultural en Chile. Tesis para optar al grado de Doctor, Universidad de Chile, Santiago de Chile, Inédito.

PAÜL I AGUSTÍ, D., 2014. Differences in the location of urban museums and their impact on urban areas. International Journal of Cultural Policy, 20(4): 471-495.

PONZINI, D., 2009. Urban implications of cultural policy networks: the case of the Mount Vernon Cultural District in Baltimore. Environment and planning C: government and policy, 27 (3): 433-450.

PRATT, M. L., 1992. Imperial Eyes: Studies in Travel Writing and Transculturation. Routledge, London.

RIUS ULLDEMOLINS, J. \& M. I. ZARLENGA, 2014. Industrias, distritos, instituciones y escenas. Tipología de clústeres culturales en Barcelona. RES, 21: 47-68.

RODRIGO MONTERO, J., 2012. De las políticas de acceso a las políticas en red Experiencias de mediación crítica y trabajo en red en museos. Revista Museos, 31: 77-87.

ROJAS GOLDSACK, J., 2014. Cultura en red: una década de teatros y centros culturales públicos 2003-2013. Consejo Nacional de la Cultura y las Artes, Santiago de Chile. 
RUBIO AROSTEGUI, J. A. \& J. RIUS ULLDEMOLINS, 2012. La modernización de la gestión pública de la cultura. Análisis comparado del caso de los equipamientos culturales de las comunidades autónomas de Cataluña y Madrid. GAPP. Revista Gestión y Análisis de Políticas Públicas, 8: 11-34.

SACCO, P. L., 2006. Il distretto culturale evoluto: competenze per l'innovazione, la crescita e l'occupazione. Goodwill, Bologna.

STROM, E., 2002. Converting pork into porcelain: cultural institutions and downtown development. Urban affairs review, 38: 3-28.

STYLIANOU-LAMBERT, T.; N. BOUKAS \& M. CHRISTODOULOUYERALI, 2014. Museums and cultural sustainability: stakeholders, forces, and cultural policies. International Journal of Cultural Policy 20 (5): 566-587.

URRUTIA FERNÁNDEZ, P., 2008. Palabras de introducción y bienvenida de la Ministra Paulina Urrutia Fernández. In: Consejo Nacional de la Cultura y las Artes, Centros Culturales. Proyección, infraestructura y gestión. Consejo Nacional de la Cultura y las Artes, Valparaíso.
VEGA LAVANDEIRA, M. DEL P. \& G. A. ZEPEDA FARIAS, 2010. Análisis del Programa de Centros Culturales del Consejo Nacional de la Cultura y las Artes: Infraestructura y Audiencias. Tesis para optar al grado de Doctor, Universidad de Chile, Santiago de Chile, Inédito.

ZARLENGA, M. I.; J. RIUS ULLDEMOLINS \& A. RODRÍGUEZ MORATÓ, 2013. Cultural clusters and social interaction dynamics: The case of Barcelona. European Urban and Regional Studies http://eur.sagepub.com/content/ early/2013/12/29/0969776413514592. abstract

ZUKIN, S., 2001. How to create a culture capital: reflections on urban markets and places. In I. Blazwick (Ed.). Century City: art and culture in the modern metrópolis (pp.259-264) Tate publishing, London. 